\title{
BMJ Open Patient experiences of co-designed rehabilitation interventions: protocol for a rapid review
}

\author{
Jonathan P McKercher (D) ,1,2 Susan C Slade (D) ,2 Jalal Jazayeri (D) ,2 \\ Anita Hodge (1) , ${ }^{3}$ Matthew Knight, ${ }^{2,4}$ Janet Green (1) ,2,3 Jeffrey Woods (I) ,,3 \\ Meg E Morris (iD) 2,3
}

\begin{abstract}
To cite: McKercher JP, Slade SC, Jazayeri J, et al. Patient experiences of co-designed rehabilitation interventions: protocol for a rapid review. BMJ Open 2022;12:e056927. doi:10.1136/ bmjopen-2021-056927

- Prepublication history and additional supplemental material for this paper are available online. To view these files, please visit the journal online (http://dx.doi.org/10.1136/ bmjopen-2021-056927)
\end{abstract}

Received 03 September 2021 Accepted 31 December 2021

Check for updates

(c) Author(s) (or their employer(s)) 2022. Re-use permitted under CC BY-NC. No commercial re-use. See rights and permissions. Published by BMJ.

${ }^{1}$ Physiotherapy, The Victorian Rehabilitation Centre, Glen Waverley, Victoria, Australia ${ }^{2}$ Academic and Research Collaborative in Health (ARCH), La Trobe University, Melbourne, Victoria, Australia

${ }^{3}$ Healthscope Limited, Melbourne, Victoria, Australia

${ }^{4}$ The Victorian Rehabilitation Centre, Glen Waverley, Victoria, Australia

Correspondence to Professor Meg E Morris; m.morris@latrobe.edu.au

\section{ABSTRACT}

Introduction Patient-centred care can be facilitated by co-design, which refers to collaboration between healthcare professionals and consumers in producing and implementing healthcare. Systematic reviews on co-design have mainly focused on the effectiveness of co-produced healthcare interventions. Less attention has been directed towards the experiences of patients in co-designed interventions. This rapid review aims to explore patient experiences of co-designed rehabilitation interventions and inform rehabilitation decision-making.

Methods and analysis A rapid review will expedite timely information on co-design experiences for stakeholders. Four electronic databases, including Cochrane CENTRAL, MEDLINE, Embase and CINAHL, will be searched for papers published from 1 January 2000 to 1 January 2022. The Cochrane Risk of Bias tool will be used for randomised trials. Critical appraisal checklists from The Joanna Briggs Institute shall evaluate the risk of bias of non-randomised trials and qualitative studies. A narrative synthesis will be provided for the quantitative studies. Thematic synthesis will be conducted on qualitative findings. The overall strength of the evidence will be measured using the Grading of Recommendations Assessment, Development and Evaluation (GRADE) framework for quantitative investigations and the GRADE-Confidence in Evidence from Reviews of Qualitative Research for qualitative studies. The results will be presented using narrative summaries, identified themes, summary tables, flow charts and quantitative statistical analyses.

Ethics and dissemination Ethics approval is not required for the review. The protocol and rapid review will be submitted to an online, open access and peerreviewed journal for publication. The review findings will be rapidly translated to consumers, clinicians, healthcare leaders, organisations, researchers and policy makers via publications, evidence summaries, conferences, workshops, websites, social media and online events. PROSPERO registration number CRD42021264547.

\section{INTRODUCTION}

Patient-centred care is integral to the delivery of high-quality healthcare and positive patient experiences. ${ }^{1}$ Facilitating patient participation across health service ecosystems is key for safe and effective patient-centred
Strengths and limitations of this study

- Co-production and co-authorship with consumers are strengths of this study.

- Timely evidence generated by using rapid review methodology will accelerate the translation of evidence into rehabilitation practice.

- Risk of bias can sometimes be introduced by rapid methods and will be controlled for by a priori recommended methods and transparent reporting of the results.

care. ${ }^{2}$ Wolf et al acknowledge The Beryl Institute definition of patient experiences as '.. the sum of all interactions, shaped by the organisation's culture, that influence patient perceptions, across the continuum of care'. Consumer participation is the gold-standard for person-centred care, and can include co-design. ${ }^{4}$ Co-design refers to collaboration between stakeholders such as patients, healthcare professionals, carers or families to design and implement therapies and services in partnership. ${ }^{5}$ Rehabilitation interventions are considered to be co-designed if a patient has participated in the planning, design or delivery, including the re-design of interventions to meet individual needs and preferences.

Rehabilitation is a person-centred approach that tailors interventions to the individual and their goals, and involves an interdisciplinary team. ${ }^{6}$ Movement rehabilitation interventions can be designed to improve mobility and independence, minimise pain and to improve a person's ability to adapt to changes in circumstances. ${ }^{6}$ Movement rehabilitation also aims to optimise movement, strength, function, upper limb control, balance and to facilitate timely discharge. ${ }^{7}$ Various methods can be used to improve patient experiences of movement rehabilitation. ${ }^{8}$ One approach is co-design, which refers to collaboration 
between healthcare professionals and consumers to design and implement therapies and services. ${ }^{5}$ Rehabilitation designed in partnership with patients is more likely to meet their needs and preferences. ${ }^{9}$ The National Institute for Health Research and other agencies across the globe have advocated co-design. ${ }^{9-12}$

Previous reviews of co-design in healthcare have centred around consumer needs; ${ }^{15101314}$ implementing co-designed interventions to influence health professional behaviours; ${ }^{5}$ evaluation of how co-design facilitates clinical and service outcomes in acute healthcare settings ${ }^{14}$ and outcomes for different co-designed hospitals tools, therapies and services. ${ }^{10} \mathrm{~A}$ new area of research is the co-design of mobile health (mHealth) interventions, also known as digital health. ${ }^{15}$ For example, one trial showed that a co-designed mHealth system supported stroke rehabilitation by improving communication of health advice and patient engagement. ${ }^{16} \mathrm{~A}$ systematic literature review by Noorbergen $e t a l^{15}$ showed that co-designed strategies were of benefit to some rehabilitation patients. The literature emphasises early co-design and there is a paucity of research on the post-design phase ${ }^{15} \mathrm{~A}$ focus on postdesign implementation may elucidate how users experience the product, service or therapy environment. ${ }^{17}$

The primary aim of this review is to explore patient experiences of co-designed interventions in rehabilitation hospitals. Secondary aims will be to understand (i) the methods used to co-design rehabilitation interventions; (ii) the ways in which co-designed rehabilitation interventions are implemented and (iii) the barriers and facilitators to implementing co-designed rehabilitation therapies. Our analysis will clarify patient experiences of the post-design implementation phases of co-designed rehabilitation.

\section{METHODS AND ANALYSIS}

Systematic reviews provide high-quality evidence syntheses to enable appraisal of policies and clinical practice. ${ }^{1819} \mathrm{~A}$ rapid review is an evidence synthesis that provides information to decision makers in a timely manner, allowing for rapid communication of research findings to endusers. $^{20}$ Components of a standard systematic review are streamlined in a rapid review, to enable fast completion and dissemination. ${ }^{21}$ Rapid reviews are particularly valuable when stakeholders have a short deadline for evidence and advice. ${ }^{19}{ }^{20}$ It is noteworthy that rapid reviews are rigorous and are not less systematic than standard systematic reviews. ${ }^{22}$ The Cochrane Rapid Review Methods Group gives recommendations on the methodology supporting rapid reviews. ${ }^{20}{ }^{21}$ These include a reduced number of reviewers for screening, streamlining data extraction and method quality appraisal processes, and restricting the inclusion criteria to a defined date range. ${ }^{20}$ They also recommend limiting the number of databases searched and minimising grey literature and supplemental searching. ${ }^{21}$
The current protocol has been published on PROSPERO, the international prospective register of systematic reviews for registration, in compliance with the Preferred Reporting Items for Systematic Reviews and MetaAnalyses Protocols (PRISMA-P) recommendations. ${ }^{23}$ In the absence of a published reporting guideline for rapid reviews, ${ }^{18}$ the protocol will be informed by the PRISMA-P guidelines and the Cochrane Rapid Review methods. ${ }^{21} 23$ The review methods will also be guided by the Cochrane Rapid Review Methods Group best practice recommendations and the PRISMA guidelines. ${ }^{21} 24$ The Enhancing Transparency in Reporting the synthesis of Qualitative research guideline will enable reporting of qualitative elements of the evidence synthesis. ${ }^{25}$

\section{Patient and public involvement}

This protocol has been co-designed and co-authored with two consumer representatives. Consumers offer an authentic lived-experience contribution, and the consumer representatives assisted in the conceptualisation of the protocol, the refinement of the research question and the editing of the manuscript. The consumers will also provide input into the evidence synthesis and assist in writing the final manuscript for the rapid review.

\section{Eligibility criteria}

Studies are to be included when they meet the following criteria: published papers in journals or conference proceedings; inclusion of participants who are adults older than 18 years; conducted in a physical rehabilitation setting, such as neuro-rehabilitation, musculoskeletal rehabilitation or cardiorespiratory rehabilitation, acute, subacute or slow stream rehabilitation; include rehabilitation interventions that are co-designed with patients; report patient experiences of co-designed rehabilitation interventions; inpatient hospital settings; empirical study design reported in English. Any study design will be included, such as randomised controlled clinical trials (RCT), non-randomised trials, cohort studies, pilot studies, feasibility analyses, single case designs, surveys and qualitative investigations.

Publications will be excluded if they pertain to drug, alcohol, vocational or psychiatric rehabilitation; relate to rehabilitation in the home or outpatient settings; are protocols, book chapters, theses, editorials, conference abstracts without an accompanying paper; are solely on paediatric or maternity participants or if they are on patient groups that require a third party to participate in the co-design process (e.g., individuals with severe cognitive impairment, dementia or delirium or those in intensive care).

\section{Identification and selection of included papers}

A health services librarian will develop the search strategies and run the electronic database searches. Four online databases (Cochrane CENTRAL, MEDLINE, Embase and CINAHL) will be searched for papers published from 1 January 2000 to 1 January 2022. Search terms for the 
following key concepts will be used: co-design; rehabilitation interventions; consumers and patients; patient experience; hospitals and acute healthcare settings. A draft example of the search strategy is given in online supplementary file 1 .

The search results will be downloaded to EndNote X9.3.1. ${ }^{26}$ The combined yield will be uploaded into Covidence to sort and select the studies against the eligibility criteria. ${ }^{27}$ Initially, the duplicates will be deleted in Covidence then titles alone will be screened for eligibility. The remaining titles with abstracts will then be screened in Covidence. At least one-quarter of the titles with abstracts will be screened by two reviewers through applying the eligibility criteria. ${ }^{20}$ The remaining titles with abstracts will be screened by one reviewer. After reaching consensus on the yield, the full texts will be obtained for the remaining abstracts. The full texts will be saved in Covidence, read in full by at least one reviewer and screened using the eligibility criteria. A second reviewer will screen the excluded abstracts and full-text studies and the two reviewers will meet to reach consensus. ${ }^{20}$ Reasons for exclusions will be noted. Any discrepancies or disagreements that arise during this process will be resolved by consultation and consensus with a third author. A PRISMA-compliant flow chart (online supplementary file 2) will record the selection process for the included studies.

\section{Method quality assessment}

Summary tables will document key elements for each investigation, such as the setting, co-design strategy, co-designed interventions and evaluation. The Cochrane Risk of Bias tool will be used to appraise the method quality for the RCTs. ${ }^{28}$ For the non-randomised trials, checklists from the Joanna Briggs Institute (JBI) critical appraisal tools will be completed to assess method quality and the risk of bias, matched to different quantitative or qualitative designs. ${ }^{29} 30$ This includes for survey and interview data, which will be summarised, tabulated and analysed for themes.

Two reviewers will assess the included studies using the Cochrane Risk of Bias tool or relevant JBI critical appraisal checklist to independently assess the trustworthiness of the included studies. ${ }^{28} 29$ The checklists include a series of questions which will help the reviewers to determine the risk of bias and the trustworthiness of the results. Each checklist has a comprehensive guide for each item. ${ }^{29} 31$

\section{Data extraction and management}

Two reviewers will independently extract the data into spreadsheets using headings such as: study design, country, first author, year, setting, sample size, participant characteristics, intervention characteristics (content, who delivered, dosage, etc), co-design strategies used, description of co-design implementation, primary and secondary outcome measures such as patient experience and effects of co-production, outcome data and results, themes, co-design barriers and co-design facilitators. The data extraction spreadsheets will be evaluated for consistency and any disagreements will be discussed and agreed on. The spreadsheets will then be combined for the data synthesis stage.

\section{Data analysis/synthesis}

Data analysis will be independently completed by two reviewers. The quantitative data will be reported according to the Synthesis without Meta-Analysis. ${ }^{32}$ Two reviewers will independently summarise and interpret the reported results for the included studies. A textual description will be provided for each study to give details on the setting, participants, intervention and findings such as effect sizes or mean changes. ${ }^{29}$

For the qualitative data, a thematic synthesis will be used within a theoretical framework of meta-synthesis and an analytical framework of thematic analysis. ${ }^{33}$ The three stages of thematic synthesis recommended by Thomas and Harden will be used for combined analysis of the primary studies. ${ }^{34}$ This includes coding the findings of the included studies and developing descriptive themes for the combined coding; identifying relationships between the descriptive themes and generating analytical themes which transcend the content of each original study. ${ }^{34}$ This synthesis approach is supported by the Cochrane Qualitative and Implementation Methods Group recommendations. They advise descriptive themes often inform policy and analytical themes inform theory. Two reviewers will independently read the included studies to code and extract the themes reported by the authors of each paper. The reviewers will then group themes according to their similarities, forming representative themes. From the consolidated themes, analytical themes will be developed independently by each reviewer and finalised by consensus. ${ }^{34}$ Summary tables will be used for the qualitative findings. Discrepancies between the two reviewers will be resolved by a third author.

\section{Confidence in cumulative evidence}

PRIMSA-P recommends that the overall strength of included studies be assessed. ${ }^{23}$ For RCTs and observational studies, the Grading of Recommendations Assessment, Development and Evaluation (GRADE) framework will be used. ${ }^{35}$ This provides a quality of evidence rating system for each review outcome. The results will be displayed in a table summarising the findings. ${ }^{35}$

Where the included studies are of a qualitative design, the strength of the findings will be measured using the GRADEConfidence in Evidence from Reviews of Qualitative Research (CERQual). ${ }^{36}$ The GRADE-CERQual is a framework for reviewers to assess the amount of confidence they can have in the review results from qualitative syntheses. ${ }^{36}$ Two reviewers (JPM, SCS) will independently perform a GRADE-CERQual assessment of the findings of each review.

\section{RESULTS}

The quantitative results will be presented as statistical analyses, summary tables, flow charts and narrative 
summaries. The qualitative results will be presented as themes and subthemes and summary tables linked to the data.

\section{DISCUSSION}

The rationale and design of a rapid review of patient experiences of co-designed rehabilitation interventions has been described. The review will identify important factors in co-production and inform optimum intervention design for movement rehabilitation.

\section{ETHICS AND DISSEMINATION}

Ethics approval will not be required for the protocol and rapid review. The protocol and accompanying review will be submitted to an international peer-reviewed journal for publication. The evidence will be rapidly translated to the research community, policy makers, consumers, health professionals and healthcare organisations using a range of implementation science methods. ${ }^{37}{ }^{38}$ We shall hold a series of consumer workshops online and face-toface, to share the results with end users. A series of digital health seminars will be conducted on the results using the Academic and Research Collaborative in Health online platform. The findings will also be presented at workshops and conferences and disseminated to health professionals at professional development seminars. An evidence summary will be posted online via social media, and on websites, to ensure that the findings have wide reach.

Acknowledgements The authors would like to thank Elizabeth Lawrence, La Trobe University librarian, for developing and conducting the search of the literature.

Contributors JPM, SCS, JJ and MEM contributed to the concept and design of the study, research question and acquisition of data and resources. JPM, SCS, JJ, AH, MK, JG, JW and MEM contributed to the planning, conduct and reporting of the work in this paper. JPM wrote the first draft of the protocol manuscript. All authors contributed to the subsequent drafts of the manuscript. The final draft was edited and approved by all authors prior to submission.

Funding This project was funded by the La Trobe University, Academic and Research Collaborative in Health.

Competing interests None declared.

Patient consent for publication Not applicable.

Provenance and peer review Not commissioned; externally peer reviewed.

Supplemental material This content has been supplied by the author(s). It has not been vetted by BMJ Publishing Group Limited (BMJ) and may not have been peer-reviewed. Any opinions or recommendations discussed are solely those of the author(s) and are not endorsed by BMJ. BMJ disclaims all liability and responsibility arising from any reliance placed on the content. Where the content includes any translated material, BMJ does not warrant the accuracy and reliability of the translations (including but not limited to local regulations, clinical guidelines, terminology, drug names and drug dosages), and is not responsible for any error and/or omissions arising from translation and adaptation or otherwise.

Open access This is an open access article distributed in accordance with the Creative Commons Attribution Non Commercial (CC BY-NC 4.0) license, which permits others to distribute, remix, adapt, build upon this work non-commercially, and license their derivative works on different terms, provided the original work is properly cited, appropriate credit is given, any changes made indicated, and the use is non-commercial. See: http://creativecommons.org/licenses/by-nc/4.0/.
ORCID iDs

Jonathan P McKercher http://orcid.org/0000-0002-8839-8353

Susan C Slade http://orcid.org/0000-0001-6325-2705

Jalal Jazayeri http://orcid.org/0000-0002-2627-9949

Anita Hodge http://orcid.org/0000-0002-5716-8811

Janet Green http://orcid.org/0000-0002-2938-6694

Jeffrey Woods http://orcid.org/0000-0001-8050-6578

Meg E Morris http://orcid.org/0000-0002-0114-4175

\section{REFERENCES}

1 Bombard Y, Baker GR, Orlando E, et al. Engaging patients to improve quality of care: a systematic review. Implement Sci 2018;13:98.

2 Merner B, Hill S, Colombo C, et al. Consumers and health providers working in partnership for the promotion of person-centred health services: a co-produced qualitative evidence synthesis. Cochrane Database Syst Rev 2019;1.

3 Wolf JA, Niederhauser V, Marshburn D. Defining patient experience. Patient Experience Journal 2014;1:7-19.

4 Australian Commission on Safety and Quality in Health Care (ACSQHC). Partnering with consumers standard, 2019. Available: https://www.safetyandquality.gov.au/standards/nsqhs-standards/ partnering-consumers-standard [Accessed Jan 2022].

5 Oakman J, Cahill LS, Clune S, et al. Effectiveness of health consumer representative involvement in implementation of interventions to change health professional behaviour. Int J Qual Health Care 2021;33:mzaa164.

6 Wade DT. What is rehabilitation? An empirical investigation leading to an evidence-based description. Clin Rehabil 2020;34:571-83.

7 World Health Organization. Rehabilitation in health systems: guide for action. Geneva World Health Organization; 2019. https://www.who. int/publications/i/item/9789241515986

8 Luxford K, Piper D, Dunbar N, Poole N. Patient-Centred care: improving quality and safety through partnerships with patients and consumers. Sydney Australian Commission on Safety and Quality in Health Care; 2011. https://www.safetyandquality.gov.au/publicationsand-resources/resource-library/patient-centred-care-improvingquality-and-safety-through-partnerships-patients-and-consumers

9 Patient Experience and Consumer Engagement. A guide to build Codesign capability. Chatswood NSW Agency for Clinical Innovation; 2019. https://aci.health.nsw.gov.au/_data/assets/pdf_file/0013/ 502240/Guide-Build-Codesign-Capability.pdf

10 Lim S, Morris H, Pizzirani B, et al. Evaluating hospital tools and services that were co-produced with patients: a rapid review. Int $J$ Qual Health Care 2020;32:231-9.

11 Wolstenholme D, Grindell C, Dearden A. A co-design approach to service improvement resulted in teams exhibiting characteristics that support innovation. Design Health 2017;1:42-58.

12 Pelton L, Knihtila M. Experience-Based Co-Design of health care services implementation guide. Boston, Massachusetts Institute for Healthcare Improvement; 2018. http://www.ihi.org/

13 Slattery P, Saeri AK, Bragge P. Research co-design in health: a rapid overview of reviews. Health Res Policy Syst 2020;18:17.

14 Clarke D, Jones F, Harris R, et al. What outcomes are associated with developing and implementing co-produced interventions in acute healthcare settings? A rapid evidence synthesis. BMJ Open 2017;7:e014650.

15 Noorbergen TJ, Adam MTP, Roxburgh M, et al. Co-design in mHealth systems development: insights from a systematic literature review. AIS Transactions on Human-Computer Interaction 2021;13:175-205.

16 Aljaroodi HM, Adam M, Chiong R. Empathic Avatars in stroke rehabilitation: a Co-designed mHealth artifact for stroke survivors. International Conference on Design Science Research in Information Systems, 2017:73-89.

17 Sanders EB-N, Stappers PJ. Probes, toolkits and prototypes: three approaches to making in codesigning. CoDesign 2014;10:5-14.

18 Stevens A, Garritty C, et al. Developing PRISMA-RR, a reporting guideline for rapid reviews of primary studies (protocol). EQUATOR Network 2018 https://www.equator-network.org/wp-content/ uploads/2018/02/PRISMA-RR-protocol.pdf

19 Higgins J, Green S, Cochrane C. Cochrane handbook for systematic reviews of interventions. Chichester, England; Hoboken, NJ: WileyBlackwell, 2008.

20 Garritty C, Gartlehner G, Nussbaumer-Streit B, et al. Cochrane rapid reviews methods group offers evidence-informed guidance to conduct rapid reviews. J Clin Epidemiol 2021;130:13-22.

21 Garritty C, Gartlehner G, Kamel C. Cochrane rapid reviews. interim guidance from the Cochrane rapid reviews methods group, 2020. Available: https://methods.cochrane.org/rapidreviews/ 
sites/methods.cochrane.org.rapidreviews/files/public/uploads/ cochrane_rr_-_guidance-23mar2020-final.pdf [Accessed Jan 2022].

22 Moons P, Goossens E, Thompson DR. Rapid reviews: the pros and cons of an accelerated review process. Eur J Cardiovasc Nurs 2021;20:515-9.

23 Moher D, Shamseer L, Clarke M, et al. Preferred reporting items for systematic review and meta-analysis protocols (PRISMA-P) 2015 statement. Syst Rev 2015;4:1.

24 Page MJ, McKenzie JE, Bossuyt PM, et al. The PRISMA 2020 statement: an updated guideline for reporting systematic reviews. BMJ 2021;372:n71.

25 Tong A, Flemming K, Mclnnes E, et al. Enhancing transparency in reporting the synthesis of qualitative research: ENTREQ. BMC Med Res Methodol 2012;12:181.

26 Endnote reference management software, Clarivate analytics. Available: www.endnote.com [Accessed Jan 2022].

27 Covidence systematic review software. Available: www.covidence. org [Accessed Jan 2022].

28 Sterne JA, Hernán MA, Reeves BC, et al. ROBINS-I: a tool for assessing risk of bias in non-randomised studies of interventions. BMJ 2016;355:i4919.

29 Aromataris E, Munn Z, eds. JBI Manual for Evidence Synthesis, 2020. https://synthesismanual.jbi.global

30 Lockwood C, Munn Z, Porritt K. Qualitative research synthesis: methodological guidance for systematic reviewers utilizing metaaggregation. Int J Evid Based Healthc 2015;13:179-87.
31 Higgins J, Savović J, Page M. Chapter 8: Assessing risk of bias in a randomized trial. In: Higgins JPT, Thomas J, Chandler J, et al, eds. Cochrane Handbook for systematic reviews of interventions version 6.2 (updated February 2021). Cochrane, 2021. www.training. cochrane.org/handbook

32 Campbell M, McKenzie JE, Sowden A, et al. Synthesis without metaanalysis (SWiM) in systematic reviews: reporting guideline. $B M J$ 2020;368:16890.

33 Noyes J, Booth A, Cargo M. Chapter 21: Qualitative evidence. In: Higgins JPT, Thomas J, Chandler J, et al, eds. Cochrane Handbook for systematic reviews of interventions version 6.2 (updated February 2021). Cochrane, 2021.

34 Thomas J, Harden A. Methods for the thematic synthesis of qualitative research in systematic reviews. BMC Med Res Methodol 2008;8:45.

35 Guyatt G, Oxman AD, Akl EA, et al. Grade guidelines: 1. IntroductionGRADE evidence profiles and summary of findings tables. J Clin Epidemiol 2011;64:383-94.

36 Lewin S, Booth A, Glenton C, et al. Applying GRADE-CERQual to qualitative evidence synthesis findings: introduction to the series. Implement Sci 2018;13:2.

37 Greenhalgh T, Papoutsi C. Spreading and scaling up innovation and improvement. BMJ 2019;365:I2068.

38 Sarkies MN, Robins LM, Jepson M, et al. Effectiveness of knowledge brokering and recommendation dissemination for influencing healthcare resource allocation decisions: a cluster randomised controlled implementation trial. PLoS Med 2021;18:e1003833. 